\title{
SEGURANÇA DA FUSÃO SELETIVA NA ESCOLIOSE IDIOPÁTICA E EVOLUÇẤO PÓS-OPERATÓRIA
}

\author{
SAFETY OF SELECTIVE FUSION AND POSTOPERATIVE EVOLUTION IN IDIOPATHIC SCOLIOSIS
}

\section{SEGURIDAD DE LA FUSIÓN SELECTIVA EN LA ESCOLIOSIS IDIOPÁTICA Y EVOLUCIÓN POSTOPERATORIA}

\section{Christiano Cruz de Andrade Lima', Fernando luiz Guedes lauda', Daniel de Abreu Oliveira², Roberto Sakamoto Falcon',} Marcos Antônio Ferreira Júnior ${ }^{1}$, Cristiano Magalhäes Menezes ${ }^{1}$

\begin{abstract}
RESUMO
Objetivos: Analisar as correções obtidas através da instrumentação segmentar seletiva torácica, utilizando parafusos pediculares, em pacientes portadores de escoliose idiopática com modificador lombar B ou C; verificar a segurança da técnica e a descompensação das curvas compensatórias não instrumentadas; observar a perda de correção das curvas instrumentadas e não instrumentadas com o tempo. Métodos: Estudo retrospectivo de pacientes portadores de escoliose idiopática com curvas 1B, 1C, 2B e 2C submetidos a tratamento cirúrgico por via posterior utilizando parafusos pediculares. As variáveis analisadas foram: sexo, idade, níveis acometidos pela escoliose, níveis instrumentados, número de parafusos pediculares utilizados, classificação segundo Lenke, ângulos de Cobb pré-operatórios coronal e sagital (incluindo inclinações), ângulos de Cobb no pós-operatório imediato e tardio e perda da correção das curvas com o tempo. Foram submetidos 20 pacientes portadores de escoliose idiopática à tratamento cirúrgico seletivo de agosto/2004 a outubro/2007. Destes, 18 permanecem em acompanhamento e foram inclú́dos no trabalho porque satisfizeram os critérios de inclusão. Resultados: A média do ângulo de Cobb no pré-operatório foi de $52,61^{\circ}$, no pós-operatório imediato foi de $17,89^{\circ}$ e no pós-operatório tardio, $22,15^{\circ}$, com média de correção imediata de $34,72^{\circ}$ e perda de correção média 4,26 em 39,78 meses. Obteve-se uma correção espontânea média imediata de $22,62^{\circ}$ nas curvas compensatórias não instrumentadas e perda média de 2,72 ${ }^{\circ}$ dessa correção em 39,78 meses. Não ocorreram complicações neurológicas, infecciosas ou descompensações das curvas lombares. Conclusão: Todos os pacientes apresentaram melhora substancial em termos estéticos, clínicos e radiológicos. Ocorreram perdas parciais da correção com o tempo, porém sem culminarem em descompensação das curvas não instrumentadas. Este trabalho mostra indícios de eficácia e segurança do tratamento cirúrgico seletivo da escoliose idiopática utilizando-se parafusos pediculares a médio/longo prazo.
\end{abstract}

Descritores: Escoliose/cirurgia; Fusão vertebral; Doenças da coluna vertebral; Curvaturas da coluna vertebral.

\begin{abstract}
Objective: To analyze the corrections obtained by selective thoracic segmental instrumentation using pedicle screws in patients with idiopathic scoliosis with lumbar B or C modificators. Determine the safety of the technique and decompensation of non-instrumented and instrumented compensatory curves. Observe the loss of the correction of instrumented and non-instrumented curves over the time. Methods: Retrospective study of patients with idiopathic scoliosis curves types $1 B, 1 C, 2 B$ and $2 C$ who underwent surgical treatment via posterior approach using pedicle screws. The variables analyzed were: sex, age, levels affected by scoliosis, instrumented levels, number of inserted pedicle screws, classification according to Lenke, pre-surgical coronal and sagital Cobb angles (inclination included), immediate and late postoperative Cobb angles and the loss of the curve correction over the time. Twenty patients with idiopathic scoliosis underwent surgical selective treatment from August 2004 to October 2007. Eighteen of these patients were followed up and were included in this study they fulfilled the inclusion criteria. Results: The average Cobb angle preoperatively was 52,610, in the immediate postoperative, it was $17,89^{\circ}$ and in the later postoperative, the Cobb angle was $22,15^{\circ}$, with an immediate mean correction of $34,72^{\circ}$ and average correction loss of $4,26^{\circ}$ at 39,78 months. An immediate average spontaneous correction of $22,62^{\circ}$ was obtained in the non-instrumented compensatory curves and an average loss of $2,72^{\circ}$ of this correction occurred at 39,78 months. There were no neurological complications, infections or decompensation of the lumbar curves. Conclusion: All patients showed substantial improvement in aesthetic terms, as well as in clinical and radiological aspects. Partial loss of correction occurred with time, but they did not lead to decompesation of non-instrumented curves. This study shows evidence of efficacy and safety of selective surgical treatment of idiopathic scoliosis using pedicle screws in the medium and long term.
\end{abstract}

Keywords: Scoliosis/surgery; Spinal fusion; Spine diseases; Spinal curvatures.

\section{RESUMEN}

Objetivos: Analizar las correcciones obtenidas a través de la instrumentación segmentar selectiva torácica, utilizando tornillos pediculares, en pacientes portadores de escoliosis idiopática con modificador lumbar B o C; verificar la seguridad de la técnica y la descompensación de las curvas compensatorias no instrumentadas; observar la pérdida de corrección de las curvas instrumentadas y no instrumentadas con el tiempo. Métodos: Estudio retrospectivo de pacientes portadores de escoliosis idiopática con curvas 1B, $1 C, 2 B$ y 2C sometidos a tratamiento quirúrgico por vía posterior utilizando tornillos pediculares. Las variables analizadas fueron: sexo, edad, niveles acometidos por la escoliosis, niveles instrumentados, número de tornillos pediculares utilizados, clasificación según Lenke, ángulos de Cobb preoperatorios coronal y sagital (incluyendo inclinaciones), ángulos de Cobb en el postoperatorio inmediato y tardío y pérdida de la corrección de las curvas

1. Hospital Ortopédico/ Life Center, Belo Horizonte, MG, Brasil.

2. Núcleo de Ortopedia eTraumatologia, Belo Horizonte, MG, Brasil.

Trabalho realizado no Hospital Ortopédico/ Life Center, Belo Horizonte, MG, Brasil.

Correspondência: Hospital Ortopédico de Belo Horizonte. Rua Professor Otávio Coelho de Magalhães, 111, Mangabeiras. 30.210-300. Belo Horizonte, MG. Brasil. cristiano@vertebral.com.br

Recebido em 25/04/2012, aceito em 03/08/2012 
con el tiempo. Fueron sometidos 20 pacientes portadores de escoliosis idiopática a tratamiento quirúrgico selectivo desde agosto/2004 a octubre/2007. De éstos, 18 permanecen en acompañamiento y fueron incluidos en el trabajo porque satisficieron los criterios de inclusión. Resultados: El promedio del ángulo de Cobb en el preoperatorio fue de 52,610, en el postoperatorio inmediato fue de $17,89^{\circ}$ y en el postoperatorio tardío, $22,15^{\circ}$, con promedio de corrección inmediata de $34,72^{\circ}$ y pérdida de corrección media de 4,26 en 39,78 meses. Se obtuvo una corrección espontánea media inmediata de $22,62^{\circ}$ en las curvas compensatorias no instrumentadas y pérdida media de $2,72^{\circ}$ de esa corrección en 39,78 meses. No ocurrieron complicaciones neurológicas, infecciosas o descompensaciones de las curvas lumbares. Conclusión: Todos los pacientes presentaron mejora sustancial en términos estéticos, clínicos y radiológicos. Ocurrieron pérdidas parciales de la corrección con el tiempo, aunque sin culminar en descompensación de las curvas no instrumentadas. Este trabajo muestra indicios de eficacia y seguridad del tratamiento quirúrgico selectivo de la escoliosis idiopática utilizándose tornillos pediculares a mediano/largo plazo.

Descriptores: Escoliosis/cirugía; Fusión vertebral; Enfermedades de la columna vertebral; Curvaturas de la columna vertebral.

\section{INTRODUÇÃO}

A escoliose idiopática é a forma mais comum de escoliose estruturada, apresentando grande prevalência nos consultórios de ortopedia. Trata-se de uma condição passível de tratamento conservador, porém em certas situações o tratamento cirúrgico se torna imperativo.

O tratamento cirúrgico da escoliose idiopática encontra-se em constante evolução. Harrington foi o primeiro a associar a instrumentação da coluna. ${ }^{1,2} \mathrm{Em} 1977$, foi introduzido o Sistema de Luque com a utilização de fios sublaminares, bilateralmente em cada segmento vertebral e sequencialmente apertados às hastes. ${ }^{3}$ A instrumentação posterior de terceira geração foi desenvolvida por Cotrel e Dubousset, ${ }^{4}$ na década de 80 , utilizando-se hastes associadas a ganchos e parafusos pediculares e seus benefícios têm sido demonstrados principalmente em relação à maior correção nos planos frontal, sagital e na rotação vertebral. ${ }^{5,6}$

Recentemente, se tem dado atenção à preservação do maior número de segmentos móveis possível, limitando-se a artrodese às curvas consideradas estruturadas. Porém, o receio de descompensação das curvas toracolombares compensatórias não instrumentadas ao longo do tempo gera desconforto nos cirurgiões em alguns casos. Não há na literatura nacional artigos que relatem a história natural pós fusão seletiva da escoliose idiopática em nossa população, tão pouco sobre o grau de perda das correções cirúrgicas e das compensatórias com o tempo.

O objetivo deste trabalho é analisar as correções obtidas imediatas e tardias, através de instrumentação segmentar seletiva das curvas torácicas, utilizando-se parafusos pediculares em pacientes portadores de escoliose idiopática, com a finalidade de avaliar o grau de perda das correções com o tempo e o aparecimento de descompensações das curvas lombares.

\section{MÉTODOS}

Foram estudados retrospectivamente, através de análise de prontuários e dos exames de imagem, os pacientes portadores de escoliose idiopática submetidos ao tratamento cirúrgico com artrodese e instrumentação posterior seletiva no período de agosto de 2004 a outubro de 2007, com acompanhamento médio de 39,78 meses (extremos 16,0 meses a 59,0 meses) até agosto de 2010. Avaliações foram realizadas no pós-operatório imediato, após 1 mês, após 6 meses e anualmente após essa última.

Utilizou-se a classificação proposta por Lenke et al. ${ }^{7}$ para orientação do procedimento cirúrgico. Através de estudos radiográficos nos planos sagital, frontal e frontal com inclinações, foram determinadas quais as curvas eram estruturadas e quais eram compensatórias, permitindo a fusão seletiva das primeiras e preservação das últimas.

Os critérios de inclusão foram pacientes portadores de escoliose idiopática com curvas tipo 1,2 da classificação de Lenke et al. ${ }^{7,8}$ e modificadores lombares B e C submetidos ao tratamento cirúrgico por via posterior com nível caudal instrumentado até ou acima de L1.

Os resultados foram analisados estatisticamente através de parâmetros radiológicos, determinando-se a magnitude das curvas pelo método de Cobb nos planos frontal e sagital pré-operatório, pós-operatório imediato e tardio, descompensações do tronco após a cirurgia e perda das correções com o tempo. Utilizou-se o programa SPSS 12.0 para tais análises através do teste "Diferença entre Médias (Teste T)".

Foram avaliados vinte pacientes submetidos à instrumentação seletiva por uma mesma equipe cirúrgica. Destes, dezoito permaneceram em acompanhamento e preenchem os critérios de inclusão no trabalho.

As características gerais dos pacientes são apresentadas na Tabela 1.

O sexo feminino representou $95 \%$ dos casos e o sexo masculino 5\%. A idade variou de 10 a 30 anos (média etária de 15,33 anos). (Figura 1)

De acordo com a classificação de Lenke et al., ${ }^{7}$ dois pacientes apresentavam curva do tipo 1B- $(11,1 \%)$, doze do tipo 1BN (66,7\%), um do tipo $1 \mathrm{CN}(5,6 \%)$, um do tipo $1 \mathrm{C}+(5,6 \%)$, um do tipo $2 \mathrm{BN}$ (5,6\%), um do tipo $2 \mathrm{CN}(5,6 \%)$. (Figura 2$)$

No pré-operatório, a média do ângulo de Cobb coronal torácico era de $52,61^{\circ}$ (extremos de $38^{\circ}$ a $68^{\circ}$ ). A média do ângulo de Cobb coronal lombar compensatório era de $35,56^{\circ}$. A média do ângulo de Cobb sagital torácico era de $21,94^{\circ}$ (extremos de $4^{\circ}$ a $41^{\circ}$ ) com dois pacientes apresentando hipocifose (ângulo de Cobb $<10^{\circ}$ ).

Tabela 1. Características gerais dos pacientes pré-operatórios.

\begin{tabular}{|c|c|c|c|c|c|c|c|c|}
\hline & ID & Sex & Ext & LENKE & $\begin{array}{c}\text { COBB } \\
\text { Torácico pré }\end{array}$ & $\begin{array}{c}\text { COBB } \\
\text { Sagital pré }\end{array}$ & RISSER & $\begin{array}{l}\text { Curvas } \\
\text { Comp pré }\end{array}$ \\
\hline 1 & 13 & $\mathrm{~F}$ & T5T12 & $1 \mathrm{~B} \mathrm{~N}$ & 50 & 20 & 5 & 29 \\
\hline 2 & 26 & $\mathrm{~F}$ & T5L1 & $1 \mathrm{BN}$ & 51 & 25 & 5 & 41 \\
\hline 3 & 14 & $F$ & $\begin{array}{l}\text { T1T6/ } \\
\text { T6T12 }\end{array}$ & $2 \mathrm{~B} \mathrm{~N}$ & $50 / 32$ & 20 & 4 & 5 \\
\hline 4 & 30 & $M$ & T5T12 & $1 \mathrm{~B}-$ & 62 & 5 & 5 & 37 \\
\hline 5 & 14 & $F$ & T4T12 & $1 \mathrm{~B} \mathrm{~N}$ & 42 & 20 & 4 & 28 \\
\hline 6 & 15 & $\mathrm{~F}$ & T6T11 & $1 \mathrm{~B} \mathrm{~N}$ & 51 & 20 & 4 & 38 \\
\hline 7 & 19 & $\mathrm{~F}$ & T5T12 & $1 \mathrm{~B} \mathrm{~N}$ & 55 & 30 & 5 & 45 \\
\hline 8 & 14 & $F$ & T6T11 & $1 \mathrm{~B} \mathrm{~N}$ & 38 & 30 & 2 & 30 \\
\hline 9 & 13 & $F$ & T5T12 & $1 \mathrm{~B}-$ & 50 & 4 & 2 & 28 \\
\hline 10 & 10 & $\mathrm{~F}$ & T5L1 & $1 \mathrm{~B} \mathrm{~N}$ & 66 & 10 & 0 & 50 \\
\hline 11 & 13 & $\mathrm{~F}$ & T5T12 & $1 \mathrm{BN}$ & 57 & 14 & 4 & 36 \\
\hline 12 & 14 & $\mathrm{~F}$ & T5T11 & $1 \mathrm{BN}$ & 54 & 12 & 3 & 34 \\
\hline 13 & 14 & $\mathrm{~F}$ & T5T12 & $1 \mathrm{BN}$ & 55 & 20 & 3 & 40 \\
\hline 14 & 12 & $F$ & T4T10 & $1 \mathrm{CN}$ & 47 & 27 & 0 & 37 \\
\hline 15 & 12 & $F$ & $\begin{array}{l}\text { T1T5/ } \\
\text { T6T12 } \\
\end{array}$ & $2 \mathrm{CN}$ & 49/68 & 36 & 2 & 51 \\
\hline 16 & 12 & $\mathrm{~F}$ & Т5Т11 & $1 \mathrm{BN}$ & 52 & 31 & 1 & 33 \\
\hline 17 & 17 & $\mathrm{~F}$ & T5T11 & $1 \mathrm{C}_{+}$ & 50 & 41 & 4 & 42 \\
\hline 18 & 14 & $F$ & T6T12 & $1 B N$ & 49 & 30 & 4 & 36 \\
\hline
\end{tabular}

$\mathrm{D}$ = idade do paciente no momento da cirurgia; $\mathrm{SEX}=$ sexo do paciente $\mathrm{F}=$ feminino $\mathrm{M}=$ masculino: EXT = extensão das curvas estruturadas na coluna vertebral; LENKE = padrão de curvas segundo no; EXT = extensão das curvas estruturadas na coluna vertebral; LENKE = padrão de curvas segundo das curvas estruturadas; COBB SAGITAL PRÉ = ângulo de Cobb no plano sagital pré-operatório; RISSER = sinal de Risser no momento da cirurgia; CURVAS COMP PRÉ = ângulo de Cobb no plano frontal pré-operatório das curvas compensatórias não estruturadas. 


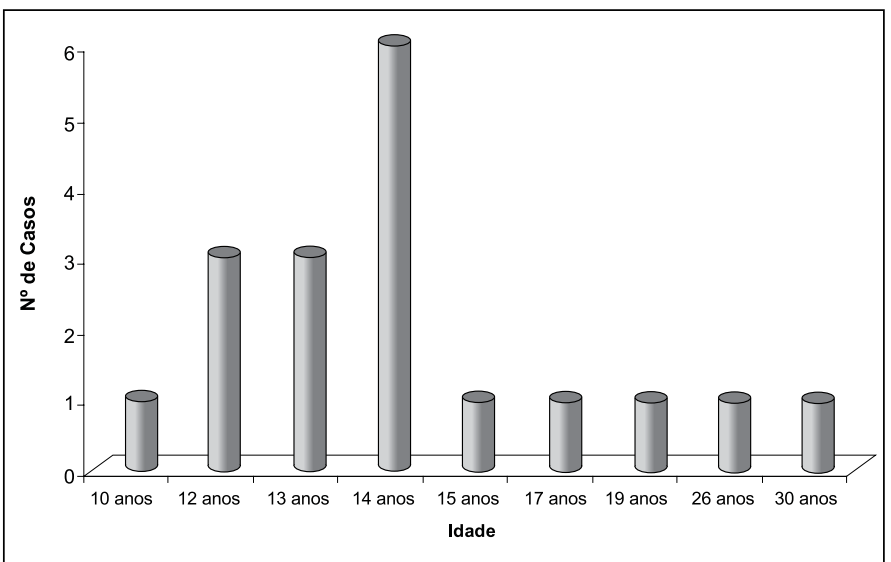

Figura 1. Número de casos por idade.

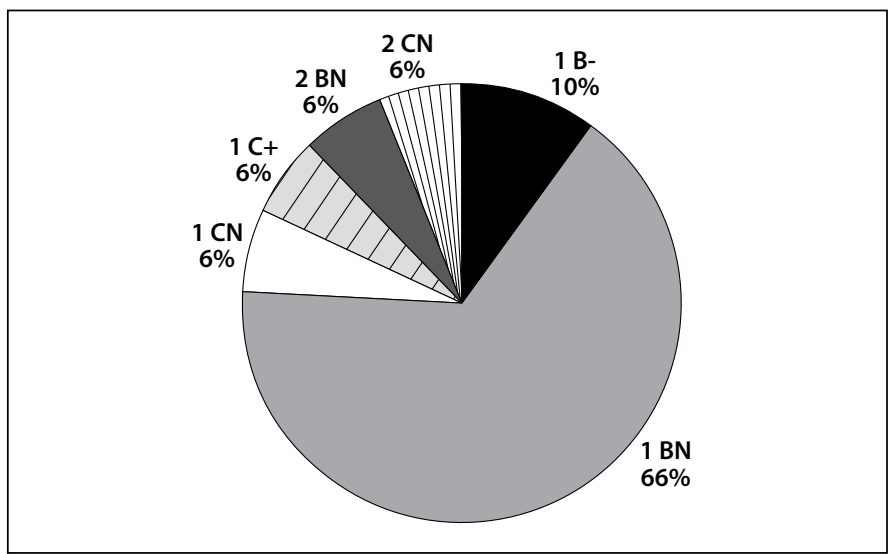

Figura 2. Porcentagem de casos por tipo de curva de Lenke.

\section{RESULTADOS}

O tempo cirúrgico médio foi de 3'59" (variação de 3'00" a 6'30") e a média do tempo de internação foi de 4 dias.

No pós-operatório imediato, a média do ângulo de Cobb torácico coronal obtido foi de $17,89^{\circ}$ (extremos de $7^{\circ}$ a $27^{\circ}$ ). A média do ângulo de Cobb torácico sagital obtido foi de $23,61^{\circ}$ (extremos de $12^{\circ}$ a $44^{\circ}$ ).

No pós-operatório tardio, a média do ângulo de Cobb torácico coronal obtido foi de $22,15^{\circ}$ (extremos de $10^{\circ}$ a $38^{\circ}$ ). A média do ângulo

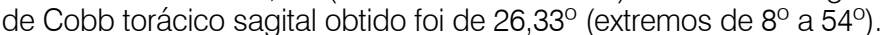

Os resultados dos ângulos de Cobb no plano sagital das curvas estruturadas pré- operatórios, pós-operatórios imediato e atual estão representados pelas Figuras 3 e 4.

A média de correção imediata do ângulo de Cobb torácico no plano coronal foi de $66,03 \%\left(34,72^{\circ}\right)$ (extremos de $28^{\circ}$ a $48^{\circ}$ ) enquanto média de correção tardia foi de 58,47\% (30.46 ) (extremos de $10^{\circ}$ a $38^{\circ}$ ), observando-se uma perda média de $4,26^{\circ} \mathrm{com} 0$ passar do tempo, estatisticamente significativa $[p=0,002]$. A correção media pré-operatória com as inclinações laterais foi de $45,7 \%$.

Conforme o padrão de curva de Lenke, as correções imediatas e tardias médias obtidas foram respectivamente de: $56,23 \%\left(24,5^{\circ}\right)$ e $51,23 \%\left(27^{\circ}\right)$ no padrão 1B-, 69,32\% $\left(15,92^{\circ}\right)$ e $62,87 \%\left(19,42^{\circ}\right)$ no padrão $1 \mathrm{BN}, 61,70 \%\left(18^{\circ}\right)$ e $70,21 \%\left(14^{\circ}\right)$ no padrão $1 \mathrm{CN}, 56 \%$ $\left(22^{\circ}\right)$ e $50 \%\left(25^{\circ}\right)$ no padrão $1 \mathrm{C}+, 56 \%\left(22^{\circ}\right)$ e $24 \%\left(38^{\circ}\right)$ no padrão 2BN, $70,59 \%\left(20^{\circ}\right)$ e $51,47 \%\left(33^{\circ}\right)$ no padrão $2 \mathrm{CN}$. (Figura 5)

Levando em consideração apenas o tipo das curvas, foi conseguida uma média de correção imediata e tardia respectivamente de $66,37 \%\left(17,50^{\circ}\right)$ e $61,07 \%\left(38^{\circ}\right)$ nas curvas tipo 1 e $63,29 \%\left(21^{\circ}\right)$ e $37,74 \%\left(31,5^{\circ}\right)$ nas curvas tipo 2. (Figura 6)

Em relação ao modificador lombar, obteve-se uma média de correção imediata e tardia de $66,69 \%\left(17,47^{\circ}\right)$ e $58,72 \%\left(21,67^{\circ}\right) \mathrm{com}$ modificador B e $62,76 \%\left(20^{\circ}\right)$ e $57,23 \%\left(24^{\circ}\right)$ com modificador C respectivamente. (Figura 7 )

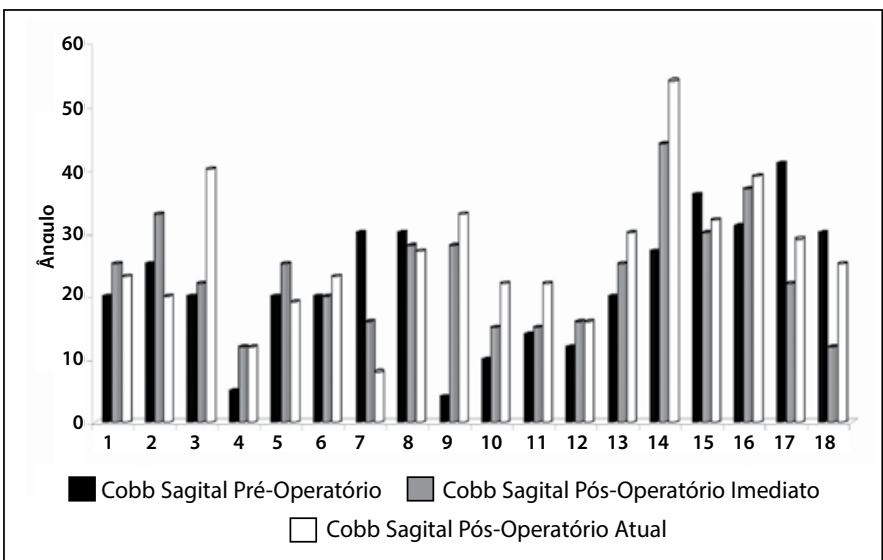

Figura 3. Ângulos de Cobb no plano sagital pré-operatórios, pós-operatório imediato e atual.

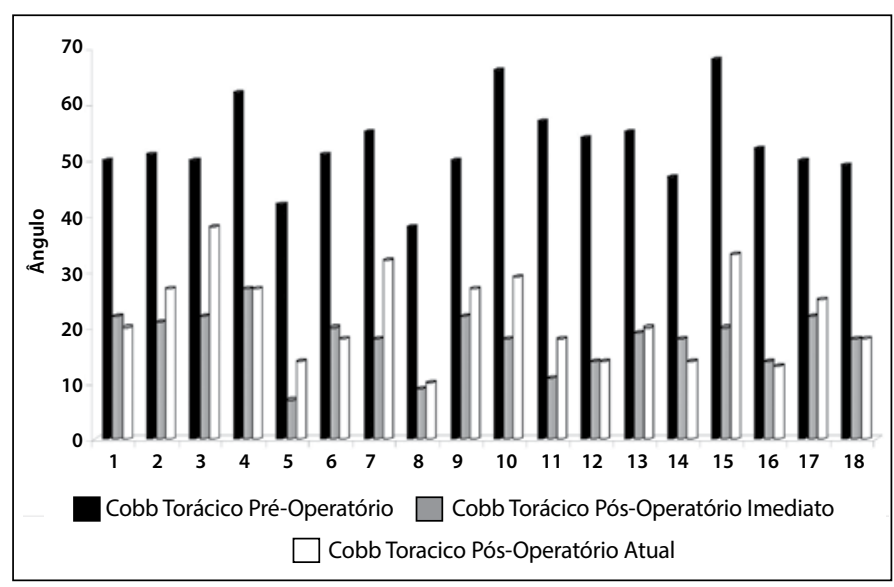

Figura 4. Ângulos de Cobb das curvas torácicas estruturadas no plano frontal pré-operatórios, pós-operatórios imediato e atual.

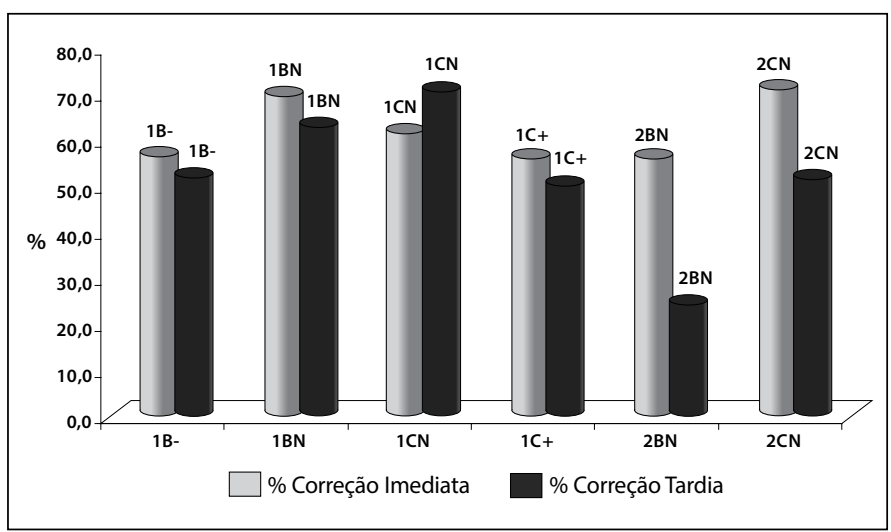

Figura 5. Porcentagem média de correção das curvas em cada padrão de curva de Lenke.

Não ocorreram lesões neurovasculares, infecções pós-operatórias ou outras complicações comuns ao procedimento como: tromboembolismo, pneumotórax, falhas do material de síntese ou pseudoartroses. Foram colocados um total de 253 parafusos pediculares. Utilizou-se em média 14 parafusos pediculares por paciente, com extremos de 11 a 19 parafusos. Ocorreu quebra do pedículo de T5 à esquerda em dois pacientes durante a passagem dos parafusos, sendo os mesmos retirados e os pedículos deixados sem instrumentação. Esses eventos não tiveram repercussões neurovasculares, tão pouco comprometeu as fixações ou o grau de correção das curvas. Com relação às curvas compensatórias lombares e toracolombares, 
foram observadas médias de correções espontâneas imediatas e tardias respectivamente de $63,38 \%\left(12.94^{\circ}\right.$ ) (extremos de $2^{\circ}$ a $22^{\circ}$ ) e $60,29 \%$ $\left(15,66^{\circ}\right)$ (extremos de $6^{\circ}$ a $25^{\circ}$ ), observando-se uma perda média de $2,72^{\circ}$ com o tempo, estatisticamente significativa $[\mathrm{p}=0,002]$.

Em relação ao modificador lombar, a média de correção imediata e tardia das curvas compensatórias foram de $66,02 \%\left(11,27^{\circ}\right)$ e $62,26 \%\left(14,40^{\circ}\right)$ com modificador B e $50,14 \%\left(21,33^{\circ}\right)$ e $50,42 \%$ $\left(21,33^{\circ}\right)$ com modificador $\mathrm{C}$ respectivamente. As medidas das curvas compensatórias pré-operatórias, pós-operatória imediatas e tardias estão representadas na Figura 8.

Com relação ao plano sagital, a cifose torácica média imediata

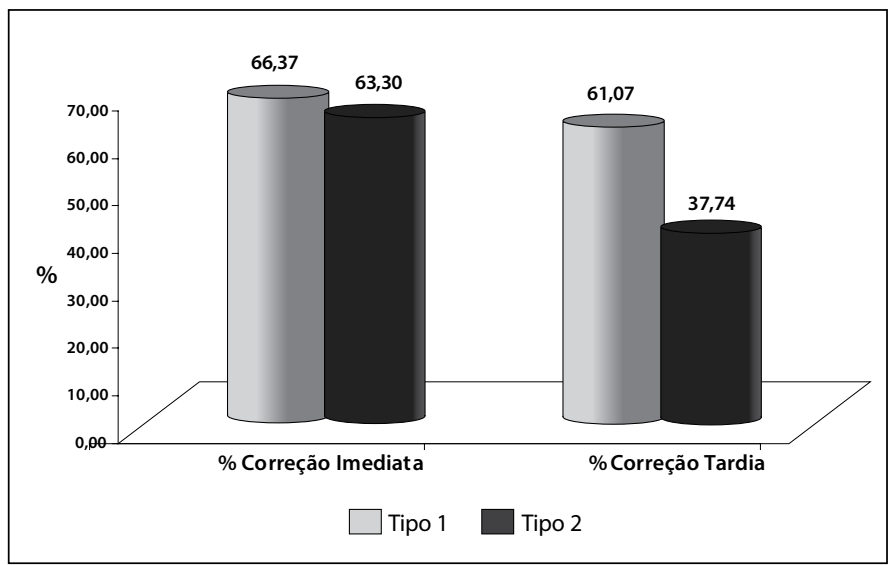

Figura 6. Porcentagem média de correção das curvas em cada tipo de curva de Lenke.

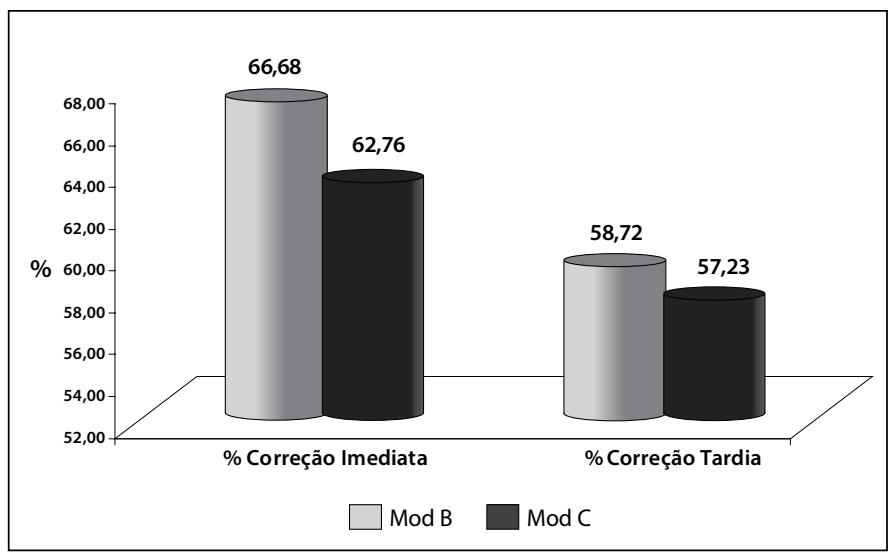

Figura 7. Porcentagem média de correção das curvas conforme o modificador lombar.

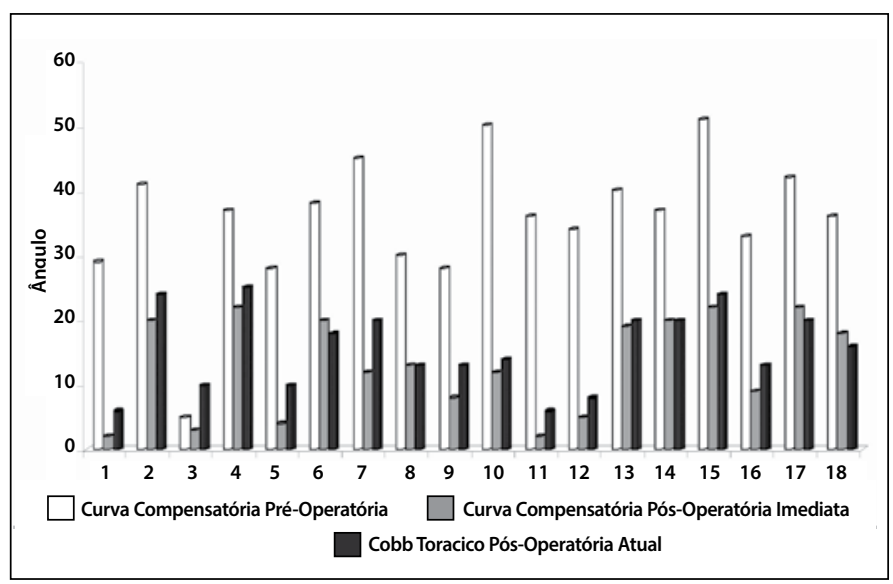

Figura 8. Ângulos das curvas compensatórias pré-operatório, pós-operatórios imediato e atual. e tardia foram de 23,61 e 26,33 graus respectivamente, sem significância estatística $[p=0,140]$.

Todos os pacientes apresentaram melhora estética importante das curvas e mostraram-se satisfeitos com o resultado, como mostrado nessas figuras anteriores. Nenhum paciente necessitou de revisão cirúrgica do procedimento. (Figuras 9-14)

Os dados pós-operatórios obtidos são apresentados na Tabela 2.
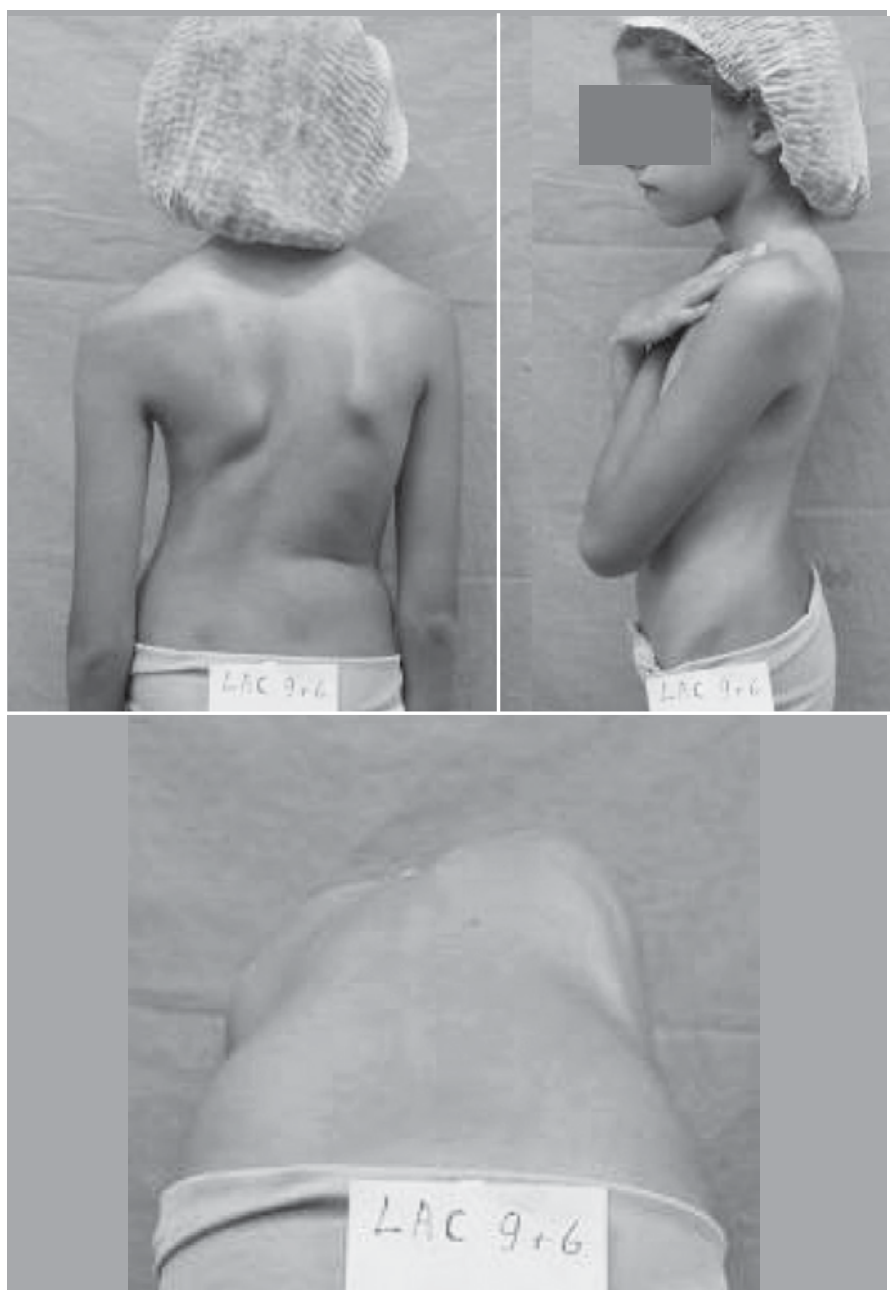

Fonte: Hospital Ortopédico, arquivo pessoal.

Figura 9. Paciente de 9 anos e 6 meses com deformidade evidente, desnivelamento de ombros, sinal do Talhe e teste de Adams +

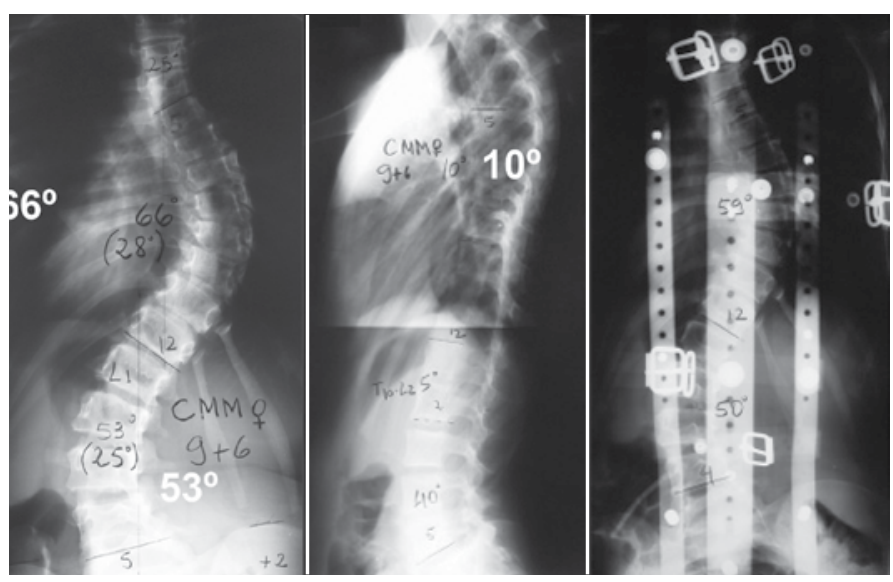

Fonte: Hospital Ortopédico, arquivo pessoal.

Figura 10. Radiografias mostrando padrão de curva 1BN de Lenke. Tentado tratamento ortótico sem sucesso. 


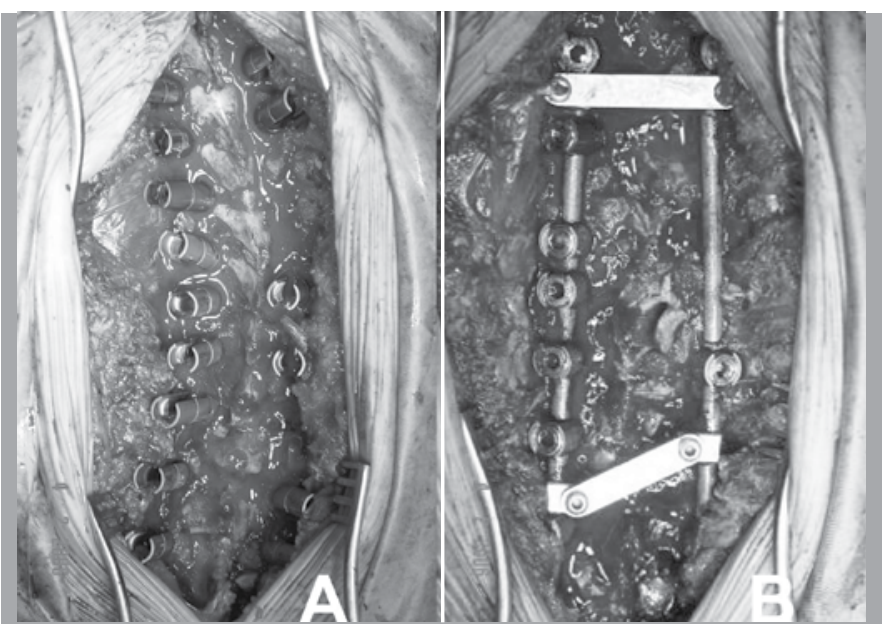

Fonte: Hospital Ortopédico, arquivo pessoal.

Figura 11. Transoperatório (A) após colocação dos parafuros pediculares e (B) correção após colocação das hastes e dos conectores

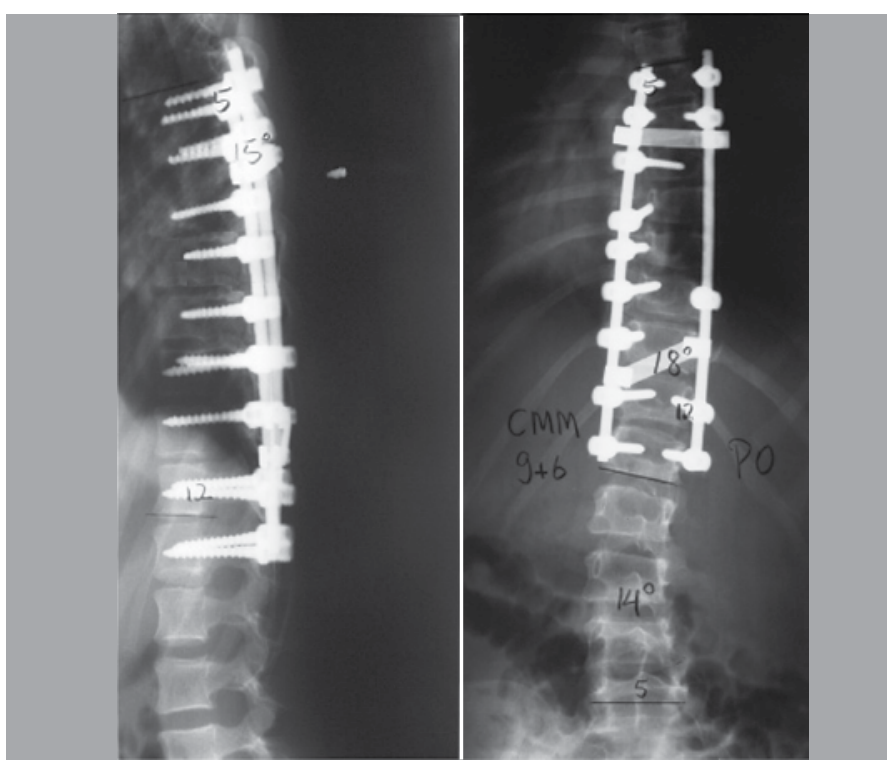

Fonte: Hospital Ortopédico, arquivo pessoal.

Figura 12. Pós-operatório imediato.

\section{DISCUSSÃO}

Lenke et al. ${ }^{7}$ descreveram uma nova classificação de escoliose que permite a avaliação tridimensional da deformidade. Trata-se de uma classificação que utiliza três componentes: tipo da curva, modificadores lombares e modificadores torácicos. Esta classificação, de 2001, considera a deformidade em dois planos, orientando o cirurgião quanto às curvas principais e compensatórias, e, portanto, quanto à abordagem mais precisa e seletiva da deformidade, o que possibilita a fusão seletiva da curva torácica, preservando a curva lombar compensatória até em pacientes com modificador lombar do tipo C. ${ }^{8-10}$ Em nossa série, foram obtidas as correções das curvas compensatórias com a artrodese seletiva apenas das curvas estruturadas, assim como proposto por Moe em 1972 apud Badra et al. ${ }^{11}$

No presente trabalho, foi utilizada a técnica cirúrgica de terceira geração, com instrumentação posterior e haste dupla, visando assim menor sangramento per operatório, menor tempo cirúrgico e menor tempo de hospitalização como proposto também por Good et al. ${ }^{12}$ em publicação de 2010.

Com o uso de parafusos pediculares, conforme demonstrado por Kim et al., ${ }^{5}$ é possível uma melhor correção da curva e melhora da função pulmonar comparando-se com a instrumentação híbrida (ganchos e parafusos).
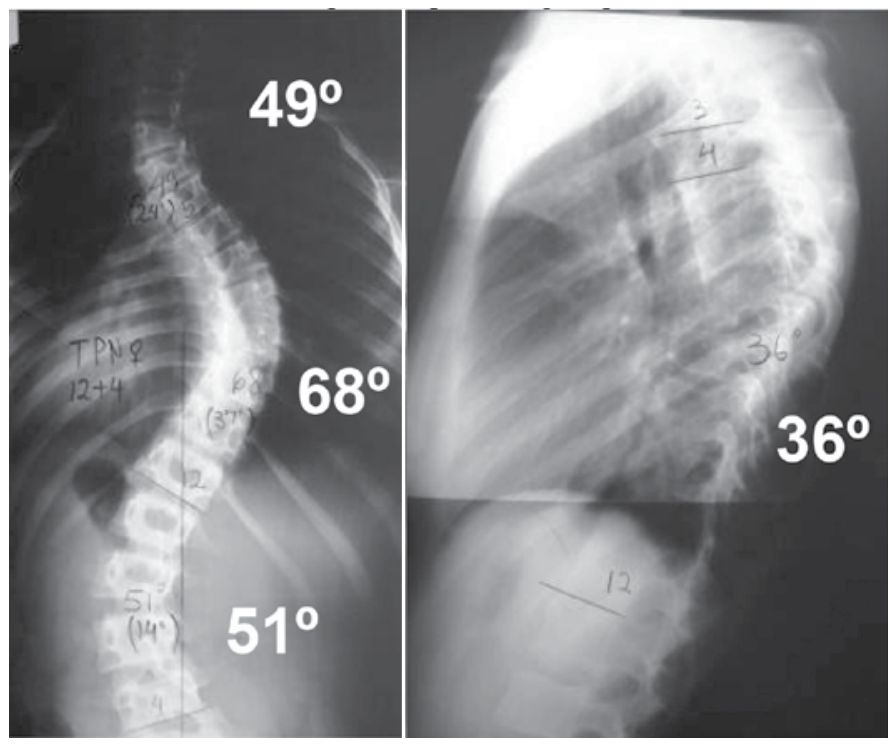

Fonte: Hospital Ortopédico, arquivo pessoal.

Figura 13. Pré-operatório de paciente de 12 anos padrão de curva $2 \mathrm{CN}$ de Lenke (Dupla torácica).
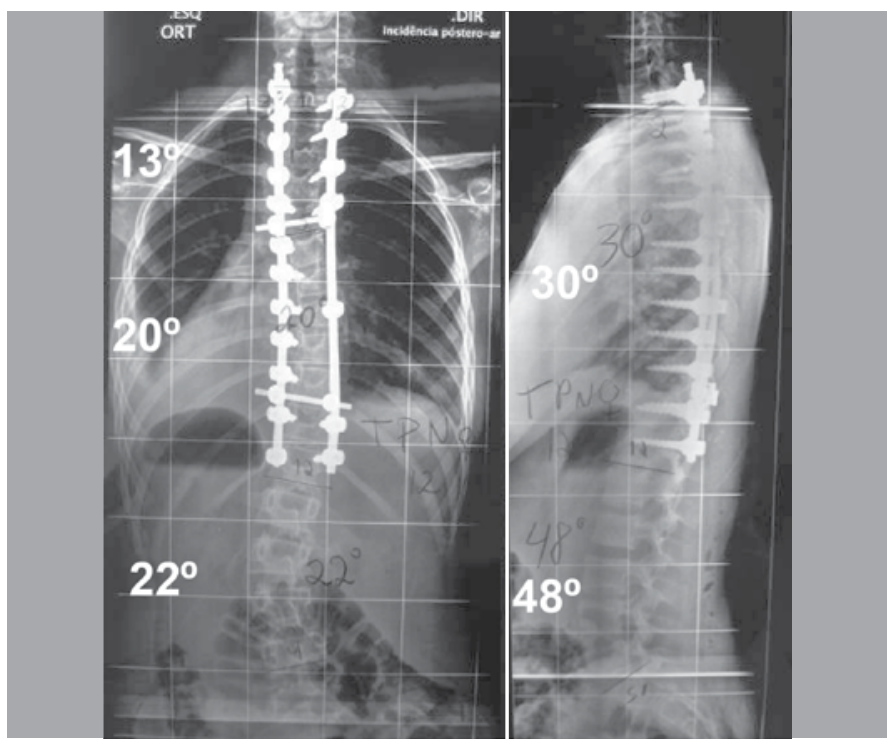

Fonte: Hospital Ortopédico, arquivo pessoal.

Figura 14. Pós-operatório imediato da mesma paciente com correção espontânea da curva compensatória lombar.

Nesta série, os graus de correção obtidos estão de acordo com a literatura que aborda o tema, ${ }^{13-15}$ sendo observados altos índices de correção das curvas no plano coronal, assim como de correção e manutenção do equilíbrio sagital, que conforme Brada et al. ${ }^{11}$ é cada vez mais reconhecido como fundamental para o tratamento cirúrgico das escolioses idiopáticas. (Tabela 2)

A manobra derrotatória é utilizada de rotina neste serviço para correção das curvas instrumentadas e não foram observadas descompensações nas curvas compensatórias decorridas dessa conduta; risco citado por Bridwell et al. ${ }^{16}$ e por Lenke et al., ${ }^{8}$ porém não observado na série de Suk et al. ${ }^{14}$

Newton et al. ${ }^{17}$ analisaram 203 pacientes com curvas de padrão 1B e 1C em um estudo multicêntrico. Dividiu-os em dois grupos: os submetidos à fusão seletiva da curva torácica e os submetidos à fusão não seletiva. Seu objetivo foi analisar as características radiográficas pré-operatórias que influenciariam os diversos cirurgiões a realizar, ou não, uma artrodese seletiva. Ele observou que $8 \%$ dos pacientes com curvas padrão $1 \mathrm{~B}$ e $32 \%$ dos pacientes com curvas 
Tabela 2. Dados pós-operatórios dos casos.

\begin{tabular}{|c|c|c|c|c|c|c|c|c|c|}
\hline & $\begin{array}{c}\text { COBB } \\
\text { Torác pós imed }\end{array}$ & $\begin{array}{c}\text { COBB } \\
\text { Sag pós imed }\end{array}$ & Instrum & Temp cir & $\begin{array}{l}\text { Temp } \\
\text { inter }\end{array}$ & $\begin{array}{c}\text { COBB } \\
\text { Cur comp pós im }\end{array}$ & $\begin{array}{c}\text { COBB } \\
\text { Torác atual }\end{array}$ & $\begin{array}{c}\text { COBB } \\
\text { Sagital atual }\end{array}$ & $\begin{array}{l}\text { COBB Curva } \\
\text { comp atual }\end{array}$ \\
\hline 2 & 21 & 33 & T5T12 (16 paraf) & $4 \mathrm{~h}$ & 3 dias & 20 & 27 & 20 & 24 \\
\hline 3 & 22 e 8 & 22 & T1T12 (13 paraf) & $4 h$ & 4 dias & 3 & $38 / 19$ & 40 & 10 \\
\hline 5 & 7 & 25 & T4T12 (14 paraf) & $4 \mathrm{~h}$ & 3 dias & 4 & 14 & 19 & 10 \\
\hline 6 & 20 & 20 & T5L1 (14 paraf) & $3 \mathrm{~h} 30 \mathrm{~min}$ & 3 dias & 20 & 18 & 23 & 18 \\
\hline 7 & 18 & 16 & T5T12 (13 paraf) & $3 \mathrm{~h} 15 \mathrm{~min}$ & 4 dias & 12 & 32 & 8 & 20 \\
\hline 8 & 9 & 28 & T6T12 (12 paraf) & 3h 30min & 4 dias & 13 & 10 & 27 & 13 \\
\hline 11 & 11 & 15 & T5L1 (14 paraf) & $3 \mathrm{~h}$ & 3 dias & 2 & 18 & 22 & 6 \\
\hline 12 & 14 & 16 & T5T12 (13 paraf) & $4 \mathrm{~h} 30 \mathrm{~min}$ & 3 dias & 5 & 14 & 16 & 8 \\
\hline 13 & 19 & 25 & T5T12 (13 paraf) & 4h 30min & 3 dias & 19 & 20 & 30 & 20 \\
\hline 14 & 18 & 44 & T4T11 (13 paraf) & $5 \mathrm{~h}$ & 3 dias & 20 & 14 & 54 & 20 \\
\hline 15 & 13 e 20 & 30 & T1T12 (19 paraf) & $6 \mathrm{~h} 30 \mathrm{~min}$ & 4 dias & 22 & $27 / 33$ & 32 & 24 \\
\hline 16 & 14 & 37 & T4T12 (15 paraf) & $3 \mathrm{~h} 30 \mathrm{~min}$ & 4 dias & 9 & 13 & 39 & 13 \\
\hline 17 & 22 & 22 & T5T12 (13 paraf) & $4 \mathrm{~h}$ & 7 dias & 22 & 25 & 29 & 20 \\
\hline
\end{tabular}

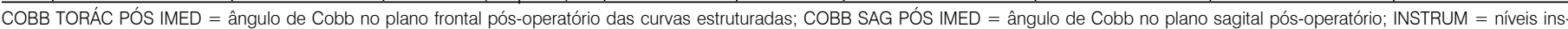

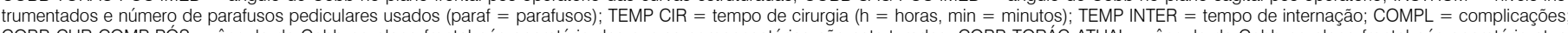

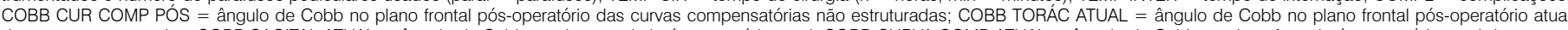

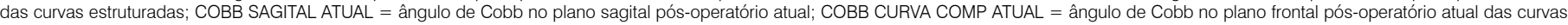
compensatórias não estruturadas.

padrão 1C foram submetidos a fusões não seletivas. Diante disso, Newton descreveu alguns aspectos que influenciavam a escolha dos cirurgiões para o uso de fusões mais extensas, dentre elas: uma curva compensatória lombar maior do que $40^{\circ}$ a $45^{\circ}$; grau de rotação vertebral dominante do ápice da curva lombar em relação ao da curva torácica; diferença entre a magnitude das curvas torácica e lombar inferior a $20 \%$, assim como uma diferença entre o desvio da linha média das mesmas curvas inferior a $20 \%$.

Em nossa série, três casos possuíam curvas compensatórias iguais ou maiores que $45^{\circ}$. Todas foram submetidas à fusão seletiva da curva torácica com cuidado especial em relação à hipercorreção e descompensação do tronco, sendo obtidos bons resultados. Ratificamos as conclusões de Newton em relação ao grau de rotação da curva lombar dominante, em relação ao da curva torácica e uma diferença entre o desvio da linha média das curvas inferior a $20 \%$ pode predispor à descompensação da curva lombar, e observamos estes aspectos de rotina.

\section{CONCLUSÃO}

Todos os dezoito pacientes apresentaram uma melhora importante do aspecto estético, clínico e radiológico com correção significativa das curvas estruturadas e compensatórias no plano coronal

\section{REFERÊNCIAS}

1. Green NE. The role of Harrington rods and Wisconsin wires in idiopathic scoliosis. In: Bridwell KH, DeWald RL, editors. The textbook of spinal surgery. 2nd ed. St. Louis: Lippincott; 1996. p. 469

2. Harrington PR. Treatment of scoliosis. Correction and internal fixation by spine instrumentation. J Bone Joint Surg Am. 1962:44:591-610.

3. Luque ER, Cardoso A. Segmental correction of scoliosis with rigid internal fixation. Orthop Trans. 1977;1:136

4. Cotrel $Y$, Dubousset J. [A new technic for segmental spinal osteosynthesis using the posterior approach]. Rev Chir Orthop Reparatrice Appar Mot. 1984;70(6):489-94.

5. Kim YJ, Lenke LG, Cho SK, Bridwell KH, Sides B, Blanke K. Comparative analysis of pedicle screw versus hook instrumentation in posterior spinal fusion of adolescent idiopathic scoliosis. Spine (Phila Pa 1976). 2004:29(18):2040-8.

6. Suk SI, Lee CK, Kim WJ, Chung YJ, Park YB. Segmental pedicle screw fixation in the treatment of thoracic idiopathic scoliosis. Spine (Phila Pa 1976). 1995;20(12):1399-405.

7. Lenke LG, Betz RR, Harms J, Bridwell KH, Clements DH, Lowe TG, et al. Adolescent idiopathic scoliosis: a new classification to determine extent of spinal arthrodesis. J Bone Joint Surg Am. 2001;83(8):1169-81.

8. Lenke LG, Edwards CC 2nd, Bridwell KH. The Lenke classification of adolescent idiopathic scoliosis: how it organizes curve patterns as a template to perform selective fusions of the spine. Spine (Phila Pa 1976). 2003;28(20):S199-207.

9. Dobbs MB, Lenke LG, Walton T, Peelle M, Della Rocca G, Steger-May K, et al. Can we predict the ultimate lumbar curve in adolescent idiopathic scoliosis patients undergoing a selective fusion with undercorrection of the thoracic curve? Spine (Phila Pa 1976). 2004;29(3):277-85 com perdas de correção aceitáveis e sem descompensações. Além disso, conseguiu-se a manutenção do equilíbrio sagital na totalidade dos casos, aspecto de suma importância devido à tendência natural da escoliose idiopática a uma hipocifose. Esse trabalho mostra indícios de eficácia e segurança do tratamento cirúrgico da escoliose idiopática, utilizando-se apenas parafusos pediculares, de forma seletiva nas curvas estruturadas a médio prazo. Não ocorreram lesões neurovasculares associadas à técnica. Porém, vale lembrar que o procedimento deve ser realizado por cirurgiões experientes, habituados com a anatomia dos pedículos vertebrais.

Apesar dos critérios quanto à previsão da evolução das curvas compensatórias não incluídas na fusão ainda não estarem totalmente definidos, seria prudente dedicar uma atenção especial àquelas que possuam grande rotação vertebral ou grande desvio do ápice em relação à linha média e realizar o máximo de esforço para preservação da cifose torácica. Deve-se esperar perdas de correção pequenas no pós-operatório tardio sem, contudo, configurar-se descompensação lombar. Estudos prospectivos com longo tempo de seguimento são necessários para a comprovação definitiva desta abordagem.

Todos os autores declaram não haver nenhum potencial conflito de interesses referente a este artigo.
10. Edwards CC 2nd, Lenke LG, Peelle M, Sides B, Rinella A, Bridwell KH. Selective thoracic fusion for adolescent idiopathic scoliosis with $\mathrm{C}$ modifier lumbar curves: 2- to 16-year radiographic and clinical results. Spine (Phila Pa 1976).2004;29(5):536-46.

11. Badra MI, Feldman DS, Hart RA. Thoracic adolescent idiopathic scoliosis: selection of fusion level. J Pediatr Orthop B. 2010:19(5):465-72

12. Good CR, Lenke LG, Bridwell KH, O'Leary PT, Pichelmann MA, Keeler KA, et al. Can posterior-only surgery provide similar radiographic and clinical results as combined anterior (thoracotomy/thoracoabdominal)/posterior approaches for adult scoliosis? Spine (Phila Pa 1976). 2010;35(2):210-8

13. Defino HLA, Fuentes ERA, Fernandes AO, Sabino Neto HS. Tratamento cirúrgico da escoliose idiopática com o uso do instrumental de Cotrel-Dubousset. Rev Bras Ortop. 2000:35(7):240-47

14. Suk SI, Lee SM, Chung ER, Kim JH, Kim SS. Selective thoracic fusion with segmental pedicle screw fixation in the treatment of thoracic idiopathic scoliosis: more than 5-year follow-up. Spine (Phila Pa 1976). 2005:30(14):1602-9.

15. Garcia E, Gonçalves R, Ribeiro G, Garcia J, Garcia L. Instrumentação de terceira geração na escoliose idiopática do adolescente. Coluna/Columna. 2006; 5(1):35-42.

16. Bridwell KH, McAllister JW, Betz RR, Huss G, Clancy M, Schoenecker PL. Coronal decompensation produced by Cotrel-Dubousset "derotation" maneuver for idiopathic right thoracic scoliosis. Spine (Phila Pa 1976). 1991;16(7):769-77.

17. Newton PO, Faro FD, Lenke LG, Betz RR, Clements DH, Lowe TG, et al. Factors involved in the decision to perform a selective versus nonselective fusion of Lenke 1B and 1C (King-Moe II) curves in adolescent idiopathic scoliosis. Spine (Phila Pa 1976). 2003;28(20):S217-23 